\title{
The natural history of pT1 colorectal cancer
}

\author{
Mauro Risio* \\ Department of Pathology, Institute for Cancer Research and Treatment, Candiolo-Torino, Italy
}

\section{Edited by:}

Michael Vieth, Klinikum Bayreuth, Germany

\section{Reviewed by:}

Lothar Veits, Klinikum Bayreuth, Germany

Helmut Neumann, University of Erlangen-Nuremberg, Germany

\section{*Correspondence:}

Mauro Risio, Department of

Pathology, Institute for Cancer

Research and Treatment, Strada

Provinciale 142, 10060

Candiolo-Torino, Italy.

e-mail:mauro.risio@ircc.it
Colorectal carcinoma invading the submucosa but not the muscular layer (pT1, early invasive cancer) represents the earliest form of clinically relevant colorectal cancer in most patients. Neoplastic invasion of the submucosa, in fact, opens the way to metastasis via the lymphatic and blood vessels, and the choice between surveillance and major surgery will turn on its metastatic potential. The following histological features predict the risk of metastasis and the different clinical outcomes: grade of differentiation of carcinoma, lymphovascular invasion, state of the resection margin. Microstaging of invasive cancer, namely the width and the depth of submucosal invasion, together with tumor budding at the advancing edge allow the metastatic risk to be further stratified in minimal, low, and high. Different, although morphologically undistinguishable, tumorigenic pathways are supposed to lead to the malignant transformation of colonic mucosa and subsequently to drive the progression from early to advanced cancer: new biomarkers are needed to identify progressive and non-progressive pT1 neoplasia.

Keywords: colon, early cancer, cancerised adenoma

\section{THE METASTATIC PHENOTYPE IN COLONIC TUMORIGENESIS}

According to the well recognized adenoma-carcinoma sequence, colorectal neoplasia is a single, indivisible continuum that, from a morphological point of view, begins within the mucosa as mild dysplasia and can progress through dysplasia of increasing severity until invasion across the muscularis mucosae occurs, at which time the term carcinoma applies (Hamilton et al., 2010). This definition of adenocarcinoma excludes diagnosis of intramucosal carcinoma in the colon or rectum, in contrast to the accepted definitions for the stomach, esophagus, and small bowel: in the latter cases, a decision on surgical versus local therapy is made based on respective protocols. Comparable lesions in the colon and rectum are reported as high grade mucosal neoplasia, because a carcinoma in the colon is defined by infiltration of the submucosa (Vieth et al., 2011), and colorectal lesions harboring "in situ" or "intramucosal" carcinoma are neither regarded nor treated as malignant ones (Figure 1).

Confirmatively, there are no known reliable examples of purely intramucosal or intraepithelial carcinomas arising in adenomas which have given rise to lymphatic metastasis, and no metastatic disease was found arising in cases of isolated, even if poorly differentiated, intramucosal colorectal carcinoma (Lewin et al., 2007).

The reason of this discrepancy has firstly to be ascribed to the paucity of lymphatic vessels in the colonic mucosa. It has been demonstrated, in fact, that no lymphatic channels can be seen in the normal mucosa at a level higher than the lowest portion of the crypt of Lieberkuhn, the vast majority of them maintaining an intimate association with the muscularis mucosae. The same relationship was found in the lamina propria of adenomatous polyps (Fenoglio et al., 1973), so that cancer cells must invade at least at the level of the submucosa in order to permeate importantly the lymphatic system. It is reasonable, however, that additional factors in the molecular machinery of the metastatic process are involved, besides the mere availability of lymphatics. In this regard, the specific interactions between E-cadherin and catenins occurring throughout the colorectal carcinogenesis can modulate the cadherin-mediated cell adhesion system (Takayama et al., 1996) and determine the potentials of invasiveness and metastasis of cancer cells in the different layers of the intestinal wall.

\section{INVASION AND PSEUDO-INVASION}

Repeated twisting of polyps, long stalked polyps above all, cause displacement of adenomatous tissue, glands together with lamina propria, through the muscularis mucosae into the submucosa. This is referred as pseudo-invasion and, occurring in $2.3-11.4 \%$ of polyps (Rossini et al., 1985), represents a major concern in the differential diagnosis with respect to invasive carcinoma, in that some patients with cancerised adenoma may require colectomy, whereas adenomas with epithelial displacement are adequately treated by endoscopic polypectomy. The presence or absence of hemosiderin, desmoplastic reaction, or lamina propria enclosing the glands are the main histological features distinguishing pseudo-invasion from true invasion (Figure 2).

Hemosiderin in the lamina propria is found in $90 \%$ of polyps with pseudo-invasion but also (though less frequently) in cases of true invasion, so that hemosiderin deposition is not a completely reliable criterion. The presence of lamina propria surrounding the glands below the muscularis mucosae is a definitive indicator of pseudo-invasion, whereas desmoplasia is a reliable criterion for true invasion. Cystic dilation of submucosal glands and masses of free mucus in the stroma are mainly attributable to mechanical displacement.

Diagnosis is more difficult when the submucosal glands display high grade dysplasia: under these circumstances, however, immunohistochemical staining for p53, E-cadherin, MMP-1, and collagen IV may be helpful (Yantiss et al., 2002). 


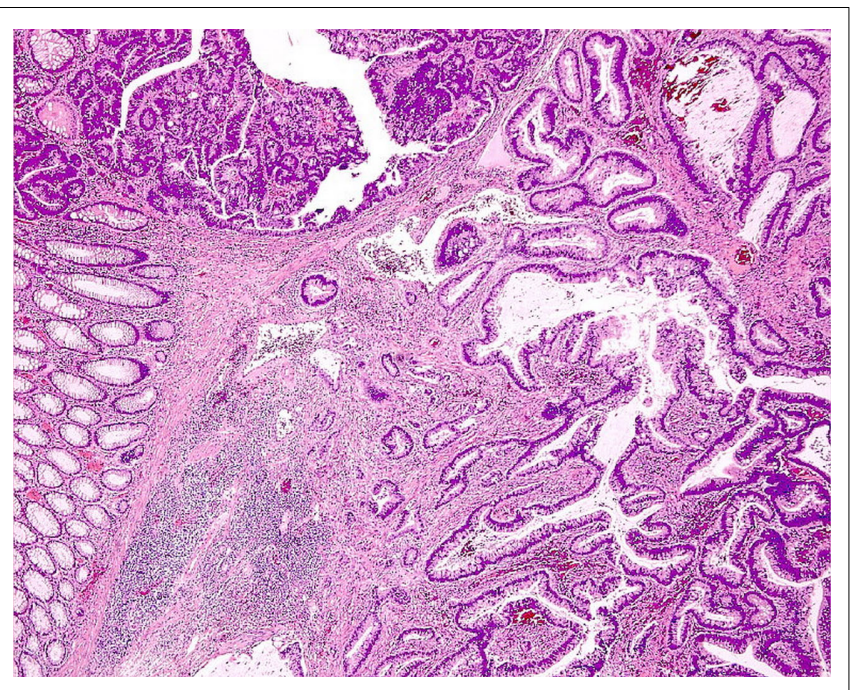

FIGURE 1 | Histologic section of a colonic adenoma containing invasive carcinoma. High grade mucosal neoplasia is strictly confined above the muscularis mucosae, whereas well differentiated adenocarcinoma extensively invades the submucosa (lower right corner).

\section{CANCERISED ADENOMAS: PATHOLOGIC PREDICTORS AND CLINICAL OUTCOMES}

Colorectal adenomas containing early invasive carcinoma comprise a carcinoma invading the submucosa but not the muscular layer (Stage pT1, according to the TNM system; Sobin et al., 2010). They are a key stage in the large bowel tumor sequence since they represent the earliest form of clinically relevant colorectal cancer in most patients. Neoplastic invasion of the submucosa, in fact, opens the way to metastasis via the lymphatic and blood vessels, and the choice between surveillance and major surgery will turn on their metastatic potential. Prevalence of pT1 cancer has been steadily rising over the past two decades, mainly due to the widespread use of colonoscopy and the improvement of polypectomy techniques, accounting for as much as $11 \%$ of all endoscopically removed polyps (Nusko et al., 1997). At present, histological parameters alone (grade of the invasive carcinoma, lymphovascular invasion, state of the resection margin) determine whether a low (7\%) or high (35\%) risk of metastasis exists (Coverlizza et al., 1989).

Although most pT1 cancers display a low grade of differentiation (well/moderately differentiated, G1-G2), $5-10 \%$ of cases, associated with a higher incidence of adverse outcomes (Cooper et al., 1998), are high grade cancers showing minimal or no tubule formation and marked cytological atypia (poorly differentiated adenocarcinoma/undifferentiated carcinoma, G3-G4; Washington et al., 2009). Tumor grade has been regarded as the most valuable predictor of lymph node involvement (Volk et al., 1995; Whitlow et al., 1997) and its evaluation is reliable when a high grade of differentiation is applied to any area of the cancer displaying poor differentiation, independently of its extension (Quirke et al., 2011).

Since cancers can spread to distant sites via the blood stream or to the regional lymph nodes, via lymphatics, definite histological

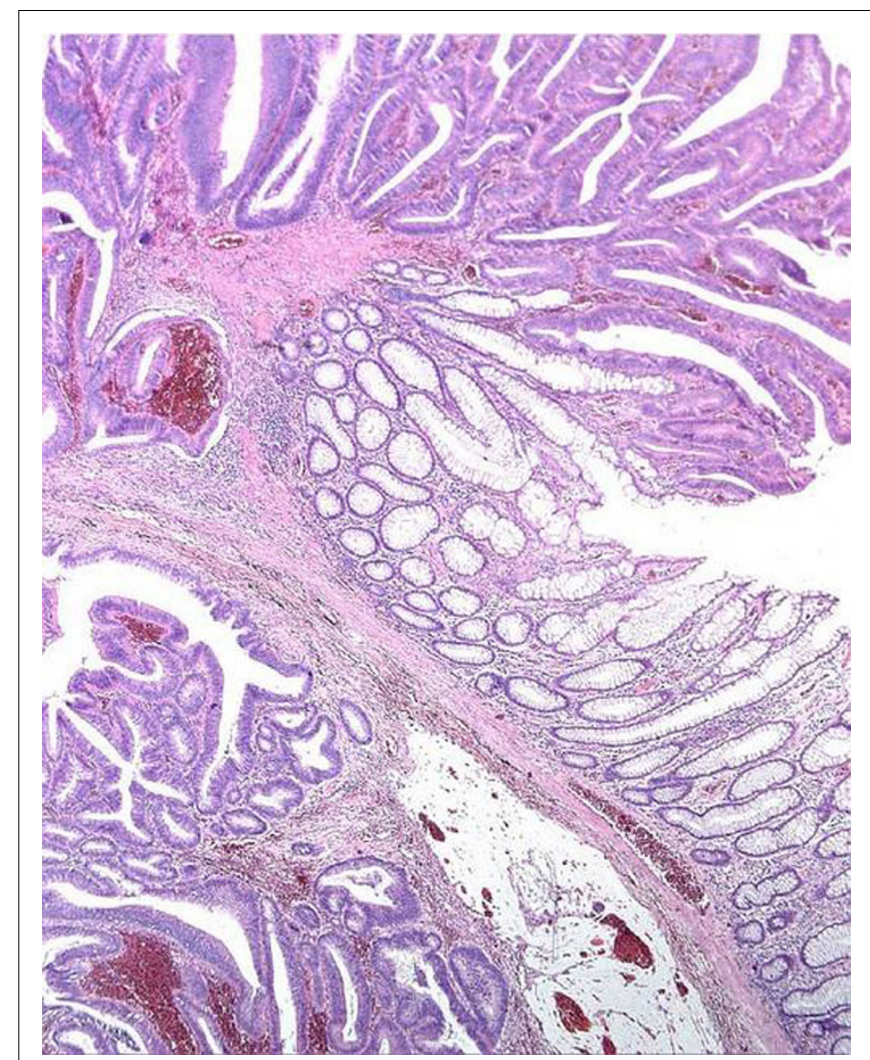

FIGURE 2 | Displacement of glands beyond the muscularis mucosae ("Pseudo-invasion"). The displaced glands are surrounded by lamina propria and close to hemosiderin deposits.

detection of lymphatic invasion or venous tumor emboli is thought to be associated with adverse outcomes. The predictive value of vascular invasion, however, has been widely debated in the literature (Jass, 1995; Kikuchi et al., 1995). Volk et al. (1995) have denied the importance of vascular invasion, that actually does not seem to be an independent risk factor at multivariate analysis (Netzer et al., 1998; Masaki and Muto, 2000). No differences in clinical outcomes were found pooling low-risk pT1 carcinomas with those with only vascular invasion (Hassan et al., 2005), although lymphatic invasion in the absence of other histologic risk factors is rarely found (Netzer et al., 1998). It has to be taken into account that distinguishing true invasion of endothelium-lined vascular spaces in the submucosa from retraction artifact around cancer cell aggregates is difficult, could require the additional use of immunohistochemistry (Ishikawa et al., 2008; Suzuki et al., 2009; Quirke et al., 2011) and impair diagnostic reproducibility (Cooper, 2007). Ueno et al. (2004), however, have demonstrated that definite vascular invasion without other unfavorable pathologic features is associated with an adverse outcome.

The resection margin obtained by endoscopic polypectomy is histologically indicated by a strip of coagulative necrosis (i.e., diathermy change) about $1 \mathrm{~mm}$ thick. The presence of cancer cells at or near the resection margin is a reliable histologic marker of adverse outcome (Cooper, 1983, 2007; Coverlizza et al., 1989). 
A negative margin is reported in the absence of cancer within the diathermy, one high-power field from diathermy, or more than $1 \mathrm{~mm}$ from the actual margin of resection (Cooper et al., 1998).

The different histological risk factors can be further linked with distinct clinical outcomes: a positive resection margin is predictive of local disease, poorly differentiated carcinoma of hematogenous metastasis and cancer-related mortality, and vascular invasion of lymph node metastasis (Hassan et al., 2005). Low- and high-risk cancers, therefore, do not only differ in lymph node metastatic potential but also - and even more importantly - as regards hematogenous metastasis, mortality rates, and overall survival (Wang et al., 2005). These observations clearly suggest that, following endoscopic polypectomy, all the histological risk factors need to be carefully evaluated by the pathologist and that the classification of patients in low- and high-risk groups is clinically meaningful.

It has been demonstrated that the level of cancer submucosal invasion is associated with the lymph node metastatic potential (Nascimbeni et al., 2002) and is important in predicting the outcome of pT1 tumors. Haggitt et al. (1985) defined the level of invasion into the stalk of the polyp for pedunculated lesions, and Kikuchi et al. (1995) the thirds of invasion (i.e., sm1, sm2, sm3) into the submucosa of non-polypoid tumors. A quantitative microstaging has also been proposed, measuring the depth and the width of submucosal invasion of pT1 cancers, that effectively predicts the metastatic potential (Ueno et al., 2004). At the present time, a firm evidence-based recommendation for one method cannot yet be made, because of lack of consensus (Quirke et al., 2011). It has to be noted, however, that the precise quantitative evaluation of the submucosal invasion depth could enable us to distinguish tumors with no or minimal risk of nodal involvement (i.e., a depth of less than $500 \mu \mathrm{m}$ ), although the proportion of tumors meeting these conditions is too small to use these categories as the criterion for a conservative approach (Ueno et al., 2004; Yasuda et al., 2007).

More recently, a unique histologic feature, tumor budding (namely the presence of scattered isolated single cells or small cluster of undifferentiated cells at the advancing edge of the cancer), has been demonstrated to be a reliable marker of the metastatic potential of cancerised adenomas. Budding describes the biological behavior of the tumor at the front of invasion in terms of epithelial - mesenchymal interactions (Prall, 2007) and its ability to predict metastases compared with the previously identified histologic factors has been proven (Hase et al., 1995; Okuyama et al., 2002; Ueno et al., 2004; Kazama et al., 2006; Sohn et al., 2007; Ishikawa et al., 2008; Ogawa et al., 2009; Suzuki et al., 2009). Despite its prognostic value, there is a great heterogeneity in reporting tumor budding, and there is no consensus on the assessment methods and cut-off values (Shinto et al., 2005; Wang et al., 2009), limiting its clinical use. However, a recent study engaging several multicentre, multinational investigators demonstrated an overall fair level of diagnostic agreement for tumor budding in colorectal cancer. In particular, diagnostic reproducibility was significantly improved in early cancer when using quantitative and selective methods of evaluation of bud cells (Puppa et al., 2012).
The balancing between morbidity and mortality associated with surgical resection and the risk of tumor progression after endoscopic polypectomy should be the main-stay of clinical decision making in pT1 cancers (Cooper et al., 2012), taking into account that post-operative mortality ranges $0.6-4.4 \%$, depending on the population, age of patient, and quality of services available (Quirke et al., 2011). While morphological markers are easily identifiable but widely ranged, biomarkers are required that are highly predictive of the events leading to the acquisition of the metastatic phenotype of early colorectal cancers. It has been previously demonstrated that, in contrast to advanced cancer, the metastatic potential of pT1 cancer is correlated neither with its proliferative activity (Risio and Rossini, 1993; Jung et al., 2001), nor with DNA ploidy (Risio et al., 1993). In the early stages, therefore, the metastatic potential is conceivable to be dependent on multiple and sequential alterations of cell-cell, cell-matrix interactions, and cell motility rather than on the derangement of cell growth. Among the adhesion molecules expressed by cancer cells, however, neither $\beta$-catenin nor claudins turned out to be related to the lymph node status (Ishikawa et al., 2008).

\section{STOCHASTIC MODELING OF EARLY CANCER}

On the whole, two distinct profiles are identifiable in the natural history of cancerised adenomas, in terms not only of lymph node metastases, but also of hematogenous spread, recurrence, and mortality. This suggests the possibility that the malignant polyp represents the end point of two different, although morphologically undistinguishable, tumorigenic, and genetic pathways, the former blocking the growth of early cancer, the latter allowing its fast progression toward advanced colorectal cancer (Hassan et al., 2005).

When malignant transformation of colonic intraepithelial neoplasia occurs, carcinoma has been thought to progress invariably, from invasion of the submucosa (pT1 stage) through the extension to the deeper structures of the intestinal wall (i.e., "advanced colorectal cancer," pT2-pT4 stages). The transition pT1-pT4 has therefore been conceived to be a continuous, progressive, irreversible process, which parallels the metastatic ratio and the decreased survival of patients (Gunderson et al., 2010). Several chromosome defects keep up with morphological evolution in colorectal tumor progression. Whilst $1 \mathrm{p}$ deletions represent an early event, numerical aberrations affecting chromosomes 7 and $18,17 \mathrm{p}$ and $18 \mathrm{q}$ deletions were reported to be the most frequent late-stage events. A stochastic model has been hypothesized, in which the transition from early to advanced stages is probabilistically regulated by the loss of subtelomeric region in $17 \mathrm{p}$ (Risio et al., 2003). Early colorectal cancer with loss of chromosome 17 actually represents the emergence from high grade dysplasia adenomatous tissue of a cell clone with genetically determined low proliferative levels, low DNA aneuploidy evolution rates and tendency to stabilize without further increase of the tumor stage. The opposite is true for pT1 colorectal cancers with $17 \mathrm{p}$ deletions, in which the invasion of the submucosa is likely to represent a fast transition toward the progressive invasion of cancer through the intestinal wall. Further research should define the biopathological features of non-progressive cancerised adenomas. 


\section{CONCLUDING REMARIS}

Cancerised adenomas (pT1 cancers) represent the earliest form of clinically relevant colorectal cancer in most patients. Invasion of the submucosa opens the way to metastasis and the choice between endoscopic surveillance and major surgery turns on their

\section{REFERENCES}

Cooper, G. S., Xu, F., Barnholtz Sloan, J. S., Koroukian, S. M., and Schluchter, M. D. (2012). Management of malignant colonic polyps: a population-based analysis of colonoscopic polypectomy versus surgery. Cancer 118, 651-659.

Cooper, H. S. (1983). Surgical pathology of endoscopically removed malignant polyps of the colon and rectum. Am. J. Surg. Pathol. 7, 613-623.

Cooper, H. S. (2007). Pathologic issues in the treatment of endoscopically removed malignant colorectal polyps. J. Natl. Compr. Canc. Netw. 5, 991-996.

Cooper, H. S., Deppisch, L. M., Kahan, E. I., Lev, R., Manley, P. N., Pascal, R. R., Qizilbash, A. H., Rickert, R. R., Silverman, J. F., and Wirman, J. A. (1998). Pathology of the malignant colorectal polyp. Hum. Pathol. 29, 15-26.

Coverlizza, S., Risio, M., Ferrari, A., Fenoglio-Preiser, C. M., and Rossini, F. P. (1989). Colorectal adenomas containing invasive carcinoma. Pathologic assessment of lymph node metastatic potential. Cancer 64, 1937-1947.

Fenoglio, C. M., Kaye, G. I., and Lane, N. (1973). Distribution of human colonic lymphatics in normal, hyperplastic, and adenomatous tissue. Its relationship to metastasis from small carcinomas in pedunculated adenomas, with two case reports. Gastroenterology 64, 51-66.

Gunderson, L. L., Jessup, M. J., Sargent, D. J., Greene, F. L., and Stewart, A. K. (2010). Revised TN categorization for rectal and colon cancers based on national survival outcome data. J. Clin. Oncol. 28, 264-271.

Haggitt, R. C., Glotzbach, R. E., Soffer, E. E., and Wruble, L. D. (1985). Prognostic factors in colorectal carcinomas arising in adenomas: implications for lesions removed by endoscopic polypectomy. Gastroenterology 89, 328-336.

Hamilton, S. R., Bosman, F. T., Boffetta, P., Ilyas, M., Morreau, H., Nakamura, S.-I., Quirke, P., Riboli, E., and Sobin, L. H. (2010). "Carcinoma of the colon and rectum," in WHO Classification of Tumours of the Digestive System, 4th Edn, eds F. T. Bosman, F.
Carneiro, R. H. Hruban, and N. D. Theise (Lyon: IARC), 134-146.

Hase, K., Shatney, C. H., Mochizuki, H., Johnson, D. L., Tamakuma, S., Vierra, M., and Trollope, M. (1995). Long-term results of curative resection of "minimally invasive" colorectal cancer. Dis. Colon Rectum 38, 19-26.

Hassan, C., Zullo, A., Risio, M., Rossini, F. P., and Morini, S. (2005). Histologic risk factors and clinical outcome in colorectal malignant polyp: a pooled-data analysis. Dis. Colon Rectum 48, 1588-1596.

Ishikawa, Y., Akishima-Fukasawa, Y., Ito, K., Akasaka, Y., Yokoo, T., Ishii, T., and The Toho Study Group for Cancer Biological Behavior. (2008). Histopathologic determinants of regional lymph node metastasis in early colorectal cancer. Cancer 112, 924-933.

Jass, J. R. (1995). Malignant colorectal polyps. Gastroenterology 109, 2034-2035

Jung, A., Schrauder, M., Oswald, U., Knoll, C., Sellberg, P., Palmqvist, R., Niedobitek, G., Brabletz, T., and Kirchner, T. (2001). The invasion front of human colorectal adenocarcinomas shows co-localization of nuclear beta-catenin, cyclin D1, P16INK4A and is a region of low proliferation. Am. J. Pathol. 159, 1613-1617.

Kazama, S., Watanabe, T., Ajioka, Y., Kanazawa, T., and Nagawa, H. (2006). Tumor budding at the deepest invasive margin correlates with lymph node metastasis in submucosal colorectal cancer detected by anticytokeratin antibody CAM5.2. Br. J. Cancer 94, 293-298.

Kikuchi, R., Takano, M., Takagi, K., Fujimoto, N., Nozaki, R., Fujiyoshi, T., and Uchida, Y. (1995). Management of early invasive colorectal cancer. Risk of recurrence and clinical guidelines. Dis. Colon Rectum 38, 1286-1295.

Lewin, R. M., Fenton, H., Burkart, A. L., Sheridan, T., Abu-Alfa, A. K., and Montgomery, E. A. (2007). Poorly differentiated colorectal carcinoma with invasion restricted to lamina propria (intramucosal carcinoma): a follow-up study of 15 cases. Am. J. Surg. Pathol. 31, 1882-1886.

metastatic potential. At present, histologic features alone are predictive of the metastatic risk and clinical outcomes. Genetic and epigenetic events drive the growth and progression of pT1 cancers, and are worthy of being intensively investigated in order to improve the management of patients with early cancer.

Masaki, T., and Muto, T. (2000). Predictive value of histology at the invasive margin in the prognosis of early invasive colorectal carcinoma. J. Gastroenterol. 35, 195-200.

Nascimbeni, R., Burgart, L. J., Nivatvongs, S., and Larson, D. R. (2002). Risk of lymph node metastasis in $\mathrm{T} 1$ carcinoma of the colon and rectum. Dis. Colon Rectum 45, 200-206.

Netzer, P., Forster, C., Biral, R. Ruchti, C., Neuweiler, J., Stauffer, E., Schonegg, R., Maure, C., Husler, J., Halter, F., and Schmassmann, A. (1998). Risk factor assessment of endoscopically removed malignant colorectal polyps. Gut 43, 669-674.

Nusko, G., Mansmann, U., Partzsch, U, Altendorf-Hofmann, A., Groitl, H., Wittekind, C., Ell, C., and Hahn, E. G. (1997). Invasive carcinoma in colorectal adenomas: multivariate analysis of patient and adenoma characteristics. Endoscopy 29 626-631.

Ogawa, T., Yoshida, T., Tsuruta, T. Tokuyama, W., Adachi, S., Kikuchi, M., Mikami, T., Saigeji, K., and Okayasu, I. (2009). Tumor budding is predictive of lymphatic involvement and lymph node metastases in submucosal invasive colorectal adenocarcinoma and non-polypoid compared with polypoid growths. Scand. J. Gastroenterol. 44, 605-614.

Okuyama, T., Oya, M., and Ishikawa, H. (2002). Budding as a risk factor for lymph node metastasis in pT1 or pT2 well-differentiated colorectal adenocarcinoma. Dis. Colon Rectum $45,628-634$.

Prall, F. (2007). Tumour budding in colorectal carcinoma. Histopathology 50, 151-162.

Puppa, G., Senore, C., Sheahan, K. Vieth, M., Lugli, A., Zlobec, I., Pecori, S., Wang, L. M., Langner, C., Mitomi, H., Nakamura, T., Watanabe, M., Ueno, H., Chasle, J., Conley, S. A., Herlin, P., Lauwers, G. Y., and Risio, M. (2012). Diagnostic reproducibility of tumour budding in colorectal cancer: a multicentre, multinational study using virtual microscopy. Histopathology. (in press).

Quirke, P., Risio, M., Lambert, R., von Karsa, L., and Vieth, M. (2011). QA in pathology in colorectal cancer screening and diagnosis - European recommendations. Virchows Arch. 458, 1-19.

Risio, M., Casorzo, L., Chiecchio, L., De Rosa, G., and Rossini, F. P. (2003). Deletions of $17 \mathrm{p}$ are associated with transition from early to advanced colorectal cancer. Cancer Genet. Cytogenet. 147, 44-49.

Risio, M., Geido, E., Rapallo, A., Pujic, N., Rossini, F. P., and Giaretti, W. (1993). DNA ploidy analysis in selected regions of colorectal adenomas containing carcinoma. Int. J. Oncol. 3, 941-947.

Risio, M., and Rossini, F. P. (1993). Cell proliferation in adenomas containing invasive carcinoma. Anticancer Res. 13, 43-48.

Rossini, F. P., Ferrari, A., Spandre, M., Scevola, F., Cavallero, M., Risio, M., Coverlizza, S. (1985). "Diagnosis and management of adenomas with invasive carcinomas: an Italian experience," in Adenomas and Adenomas Containing Invasive Carcinomas of the Large Bowel, eds C. M. Fenoglio and F. P. Rossini (New York: Raven Press), 83-102.

Shinto, E., Mochizuki, H., Ueno, H., Matsubara, O., and Jass, J. R. (2005) A novel classification of tumour budding in colorectal cancer based on the presence of cytoplasmic pseudo-fragments around budding foci. Histopathology 47, 25-31.

Sobin, L. H., Gospodarowicz, M. K., and Wittekind, Ch. (2010). TNM Classification of Malignant Tumors, 7th Edn. Chichester: Blackwell Publishing Ltd.

Sohn, D. K., Chang, H. J., Park, J. W., Choi, D. H., Han, K. S., Hong, C. W., Jung, K. H., Kim, D. Y., Lim, S.-B., Choi, H. S., and Jeong, S.-Y. (2007). Histopathological risk factors for lymph node metastasis in submucosal invasive colorectal carcinoma of pedunculated or semipedunculated type. J. Clin. Pathol. 60, 912-915.

Suzuki, A., Togashi, K., Nokubi, M., Koinuma, K., Miyakura, Y., Horie, H., Lefor, A. T., and Yasuda, Y. (2009). Evaluation of venous invasion by elastica van Gieson stain and tumor budding predicts local and distant metastases in patients with $\mathrm{T} 1$ stage colorectal cancer. Am. J. Surg. Pathol. 33, 1601-1607. 
Takayama, T., Shiozaki, H., Shibamoto, S., Oka, H., Kimura, Y., Tamura, S., Inoue, M., Monden, T., Ito, F., and Monden, M. (1996). $\beta$-Catenin expression in human cancers. Am. J. Pathol. 148, 39-46.

Ueno, H., Mochizuki, H., Hashiguchi, Y., Shimazaki, H., Aida, S., Hase, K., Matsukuma, S., Kanai, T., Kurihara, H., Ozawa, K., Yoshimura, K., and Bekku, S. (2004). Risk factors for an adverse outcome in early invasive colorectal carcinoma. Gastroenterology 127, 385-394.

Vieth, M., Quirke, P., Lambert, R., von Karsa, L., and Risio, M. (2011). Annex to Quirke et al. Quality assurance in pathology in colorectal cancer screening and diagnosis: annotations of colorectal lesions. Virchows Arch. 458, 21-30.

Volk, E. E., Goldblum, J. R., Petras, R. E., Carey, W. D., and Fazio, W. W. (1995). Management and outcome of patients with invasive carcinoma arising in colorectal polyps. Gastroenterology 109, 1801-1807.

Wang, H. S., Liang, W. Y., Lin, T. C., Chen, W. S., Jiang, J. K., Yang, S. H., Chang, S. C., and Lin, J. K. (2005). Curative resection of T1 colorectal carcinoma: risk of lymph node metastasis and long-term prognosis. Dis. Colon Rectum 48, 1182-1192.

Wang, L. M., Kevans, D., Mulcahy, H., O'Sullivan, J., Fennelly, D., Hyland, J., O'Donoghue, D., and Sheahan, K. (2009). Tumor budding is a strong and reproducible prognostic marker in T3N0 colorectal cancer. Am. J. Surg. Pathol. 33, 134-141.

Washington, M. K., Berlin, J., Branton, P., Burgart, L. J., Carter, D. K., Fitzgibbons, P. L., Halling, K., Frankel, W., Jessup, J., Kakar, S., Minsky, B., Nakhleh, R., Compton, C. C. (2009). Protocol for the examination of specimens from patients with primary carcinoma of the colon and rectum. Arch. Pathol. Lab. Med. 133, 1539-1551.

Whitlow, C., Gathright, J. B., Hebert, S. J., Beck, D. E., Opelka, F G., Timmcke, A. E., and Hicks, T. C. (1997). Long-term survival after treatment of malignant colonic polyps. Dis. Colon Rectum 40, 929-934.

Yantiss, R. K., Bosenberg, M. W., Antonioli, D. A., and Odze, R. D. (2002). Utility of MMP-1, p53, E-Cadherin, and Collagen IV immunohistochemical stains in differential diagnosis of adenomas with misplaced epithelium versus adenomas with invasive adenocarcinoma. Am. J. Surg. Pathol. 26, 206-215.

Yasuda, K., Inomata, M., Shiromizu, A., Shiraishi, N., Higashi, H., and Kitano, S. (2007). Risk factors for occult lymph node metastasis of colorectal cancer invading the submucosa and indications for endoscopic mucosal resection. Dis. Colon Rectum 50, 1370-1376.
Conflict of Interest Statement: The author declares that the research was conducted in the absence of any commercial or financial relationships that could be construed as a potential conflict of interest.

Received: 16 January 2012; paper pending published: 06 February 2012; accepted: 19 February 2012; published online: 05 March 2012.

Citation: Risio M (2012) The natural history of pT1 colorectal cancer. Front. Oncol. 2:22. doi: 10.3389/fonc.2012.00022

This article was submitted to Frontiers in Gastrointestinal Cancers, a specialty of Frontiers in Oncology.

Copyright (c) 2012 Risio. This is an openaccess article distributed under the terms of the Creative Commons Attribution Non Commercial License, which permits non-commercial use, distribution, and reproduction in other forums, provided the original authors and source are credited. 